\title{
Performance Evaluation of a Network Using Simulation Tools or Packet Tracer
}

\author{
Sayed Mansoor Hashimi, Ali Güneş
}

\begin{abstract}
Today, the importance of information and accessing information is increasing rapidly. With the advancement of technology, one of the greatest means of achieving knowledge are, computers have entered in many areas of our lives. But the most important of them are the communication fields. This study will be a practical guide for understanding how to assemble and analyze various parameters in network performance evaluation and when designing a network what is necessary to looking for to remove the consequences of degrading performance. Therefore, what can you do in a network performance evaluation using simulation tools such as Network Simulation or Packet tracer and how various parameters can be brought together successfully? CCNA, CCNP, HCNA and HCNP educational level has been used and important setting has been simulated one by one. At the result this is a good guide for a local or wide area network. Finally, the performance issues precautions described. Considering the necessary parameters, imaginary networks were designed and evaluated both in CISCO Packet Tracer and Huawei's eNSP simulation program. But it should not be left unsaid that the networks have been designed and evaluated in free virtual environments, not in a real laboratory. Therefore, it is impossible to make actual performance appraisal and output as there is no actual data available.
\end{abstract}

Keywords: Router, Network Performance Evaluation, eNSP, Packet Tracer

\section{Introduction}

Network performance evaluation with simulation program has been the subject of much research over the past years. However, the impact of performance on a network's users is much less understood from a scientific standpoint.This study will be a practical guide for understanding how to assemble and analyze various parameters in the evaluation of network performance, and what to consider when designing a network to remove the causes that reduce performance as a result.the configuration of network which has been done as a virtual in simulation program can be done in real area of network configuration. Those who complete these trainings can configure the LAN, WAN, WLAN and WWAN network design, configuration of active network devices such as router and switch, network optimization and performance settings, and can also manage the settings if necessary. This leads to network configuration greater profits for the enterprise each level that uses the network service. In turn, the increased profits, justify current and future investments in improving the performance network of the services.

Introducing the Large distributed network services are at core of a wide range of network specialist activities in the field of networking. Billions of clients and users routinely interact with networks of various kinds for different commerce, news, entertainment, and social networking. Computer Networks are usually designed with a conclusion of performance in mind [1].

\section{Performance Testing}

\section{Difference between Performance Testing, Load Testing and Stress Testing}

While connecting a device with the network to send or receive data from a specific resource to a specific destination. The significant matter is speed of network performances that how fast the data is going from one node to another. To recognize and determine the component parts of a system performance and how networks are performing in a particular situation, usage of resources, reliability and validity of the product is under testing of subtest of performance engineering. Focusing on each nodes addressing performance issues in the design, architecture of software products.

Performance testing goal: The ultimate goal for performance testing is to sit a standard benchmark behavior to the network performance. Many industries defined benchmark that should meet during performance testing. Performance testing does not aim to address and find the defect in the application of performance, but it's addressing more critical task of testing the benchmark and standards of the application, validity and accuracy and monitoring of the performance is the primary goal of performance testing.

Example: Testing the performance of network on connection speed vs latency chart, latency is the time difference between data to reach from source to destination. So, A $65 \mathrm{~Kb}$ Page would take about less 15 second to load in for a worst connection of $28.8 \mathrm{kbps}$ modem, in other hand same pages will appear within 5 second 
with average connection of $256 \mathrm{kbps}$ DSL (Latency $=100$ millisecond) $1.5 \mathrm{mbps}$ T1 would have their performance benchmark set within 1 second to achieve the target. The time difference between the generation of request and acknowledgement of response should be in the range of x ms (milliseconds) and y ms, where $\mathrm{x}$ and $\mathrm{y}$ are standard digits. A successful performance testing should project most of the performance issues, which could be related to database, network, software, hardware etc [2].

\section{Load Testing}

Load testing job is to test the system gradually and sustain to add the load of system until the time get the start point. It is the easiest method of testing to use automation tools, for instance, LoadRunner or any suited system tools, those we have already. Load testing is known as volume testing and endurance testing which are really worldwide names for load testing. The critical aim of load testing is to give a job for system to most main jobs which can possibly control the test and get their outcome through endurance and monitoring. The marvelous fact is that sometimes the result of the system is zero, it is because of the system is sometimes nourished with empty task in order to determine the behavior and reaction.

Load Testing Goal: The targets of load testing are to show and uncover the weaknesses and mistakes in application, such as memory problems, memory trickles and misconduct of memory. Another aim of load testing is to know the top limit of the whole components of application as network, database and hardware etc...thus it will be able to control and manage the estimated load in upcoming activities. Load balancing dilemmas, squad width problems, capability of current system and so on are the things that would finally figure out as a result of load testing [3].

Example: For instance, more than 1000 people will be busy with their email at same time, when you want to check email functionality of each application transactions, some users will be replying, some will be sending, deleting, forward, read and so on, in various ways. If we suppose a single transaction per person each hour, then we will have 1000 transactions in one hour, by pretending 10 transactions per user, we are able to load test the email server with surrounding it with 10000 transactions per hour.

\section{Stress Testing}

Testing under stress, different tasks to load more the current resources with better jobs are known which have effort to make the system down. Negative testing, which contain termination of one aspect or part from system and also known as component of stress testing. Furthermore, is named as fatigue testing, as we know that fatigue is mostly in exercise but here we can call stress testing as fatigue testing, this testing must take the maintaining of the application by testing it instead of its bandwidth capability. The aim of stress testing is to find out the error of system and to control how the system discover and recover back well. Before they launch the test anywhere they had a challenge that to set up a monitored environment that can exactly take the behavior of system again and again, under stress testing is mostly unprosecuted situations.

Stress Testing Goal: The target of stress testing is to examine post-smash reports to express the attitude of application after failed one. The hugest problem is to make sure that the system does not negotiate with the safety of important data after the failed one. In a good stress testing, the system will be back to the first condition along with all its parts, when the most awful situation break down after that [4].

\section{Cisco Packet Tracer}

Almost all network specialists have network simulators that help them. Because it is not always possible to work in the laboratory. And these simulation programs is very good for network configuration practice and after that easily can configure network in a real area, these simulators are such as NS (Network simulator) Cisco Packet Tracer and eNSP. Practicing the network configuration in a real network laboratory requires a lot of bagdage and times. This study has focused mainly on network performance evaluation and the configuration of network on eNSP and Cisco simulation programs. The Cisco Packet Tracer and eNSP program is a simulation program that provides a network lab environment for users to perform Cisco operations or applications without requiring the use of any physical machine or vehicle. With this software developed by Cisco, networks can be modeled virtually and become very easy to process. These Cisco programs run on virtually any platform and are user-friendly, making them simple to set up and use. The Cisco Packet Tracer has a simple interface (Figure 1), which allows your creation to perform operations by simply dragging and dropping the topology and entering the packet metric Interfaces can be easily referenced as desired on devices. all operations can be done on the network. [5] 


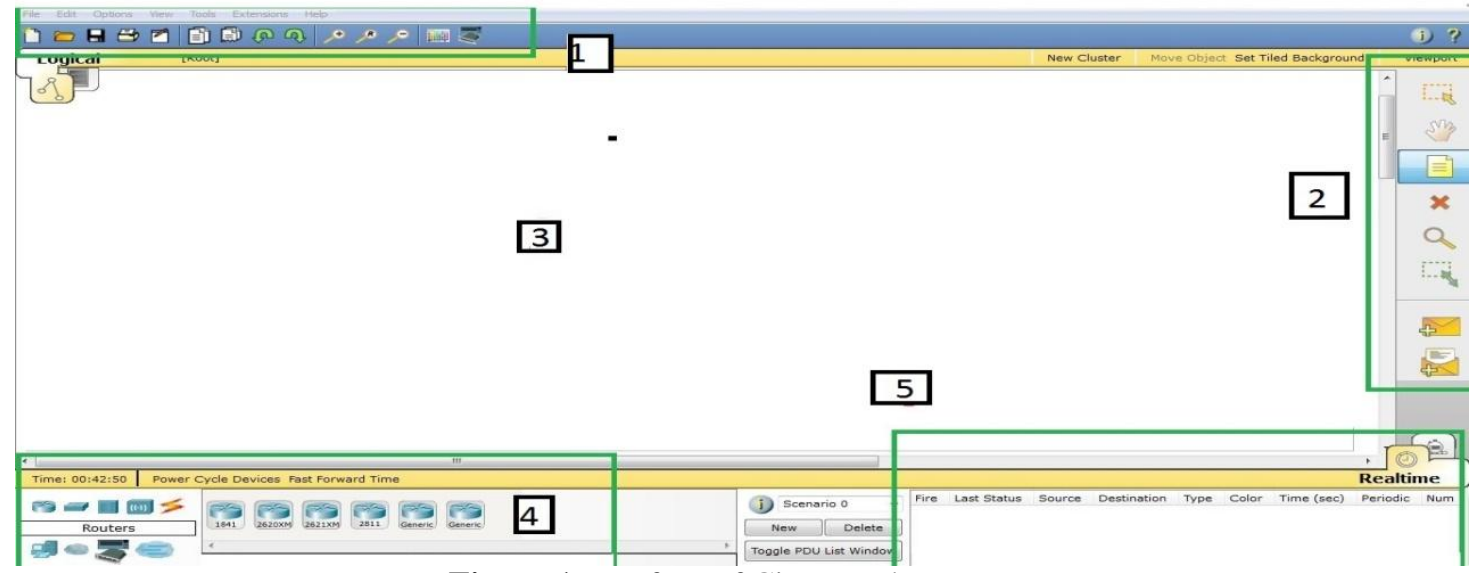

Figure 1 Interface of Cisco Packet Tracer

Nowadays, the Cisco Packet Tracer interface is so simple that you do not even need to write a ping command to see if a network device is working, it allows you to ping through an envelope-based device on the device so you can understand the status of the device.

\section{Simulation}

In this study, the Packet Tracer which can perform network performance measurement and network laboratory operations by the simulation drawing and the cisco system is made through the net simulation program. It is drawn in details in Figure 5 as it will be seen as the basic network. There is three-part scheme, An ISP and routers, modem and computers are used. While the cables connected to the router are fiber optic, other twisted pairs are preferred. Serial ports and router ip addresses are manually entered and all other are statically configured. Fast Ethernet is used for ingress and the IP addresses and MAC addresses are matched as given [6]. In addition, in this study many scenarios have been simulated. The devices, which are used in the network, provide information about the cabling local area network topology, and Package follower environment software included in this simulation Data transfer methods are divided into 3: single point, multipoint, and multiple transfers. Multiple addresses must be sent to multiple addresses for a single destination address transfer the broadcast is transmitted to all nodes in the network's dataset. All these transmissions are sent in one package. As a result, and the data will always be transmitted over all the networks. Figure 2 represents the network measurement which has 3 main parts: office, home, and central office. The cable from the modem is shared between the wireless modem. Simulation experiments have proven that the network topology works and preforming is done correctly.

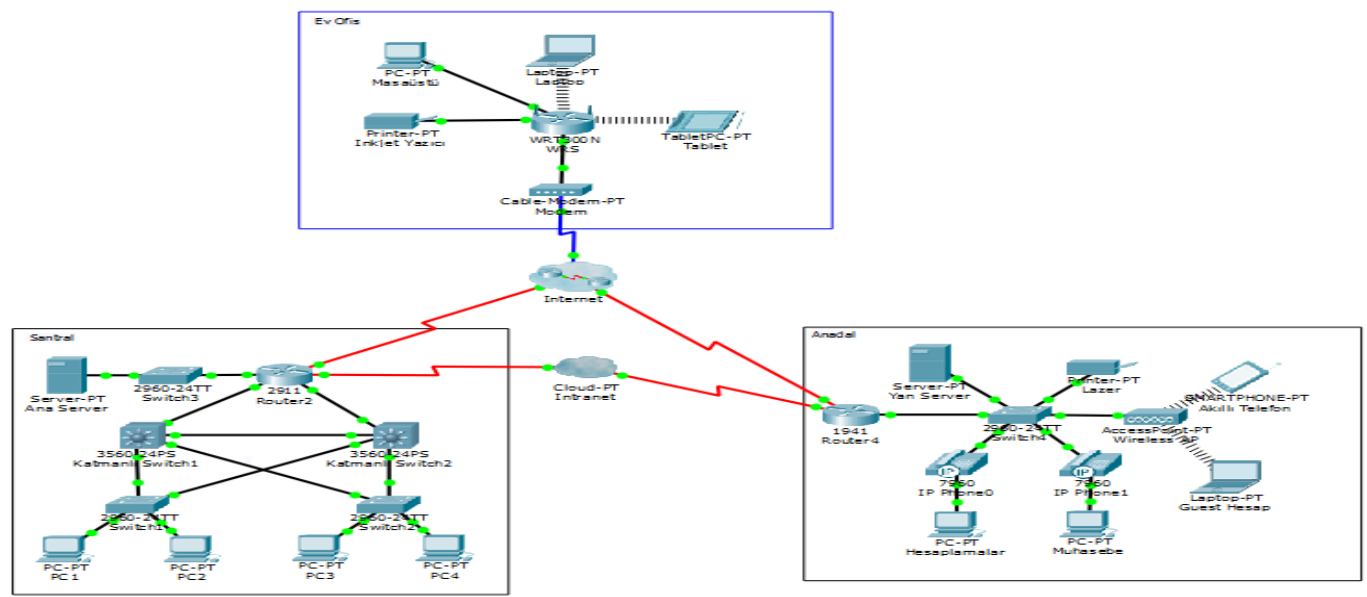

Figure 2 Sample of 3 network (office, home and central office) measurement

When designing network topologies and configuring networks we need for software that will test what we will do while working with network systems, there are many simulator programs to get rid of those needs. When we operate, for configuration of our network eNSP is used as a Simulator program. (ENSP) or Enterprise Network Simulation Platform is extensible, free and graphic network simulation platform developed by Huawei Company. By simulating Huawei enterprise switches and routers, it demonstrates network devices deployment 
scenarios. ENSP can simulate small and large-sized networks. Users and clients can perform trial tests of network and performing and learn network technologies without using real devices. We can download free eNSP simulation program from internet and the setup time firewall must be turned on because the Router Switch, Access Point, Access Controller, Access Controller, Firewall and Cloud Engine devices must be allowed to operate each of them individually. When installing this program, Wire shark Win cap, and Oracle VM Virtual Box software, is also installing together [7].

\section{Network Designing Configuration}

In designing of network which has been done in eNSP simulation program has consists of 5 routers, 9 switches, 2 access controllers, 12 wired computers, 6 laptop computers, 2 mobile phones, DHCP (Dynamic Host Configuration Protocol) and DNS (Domain Name System) servers.

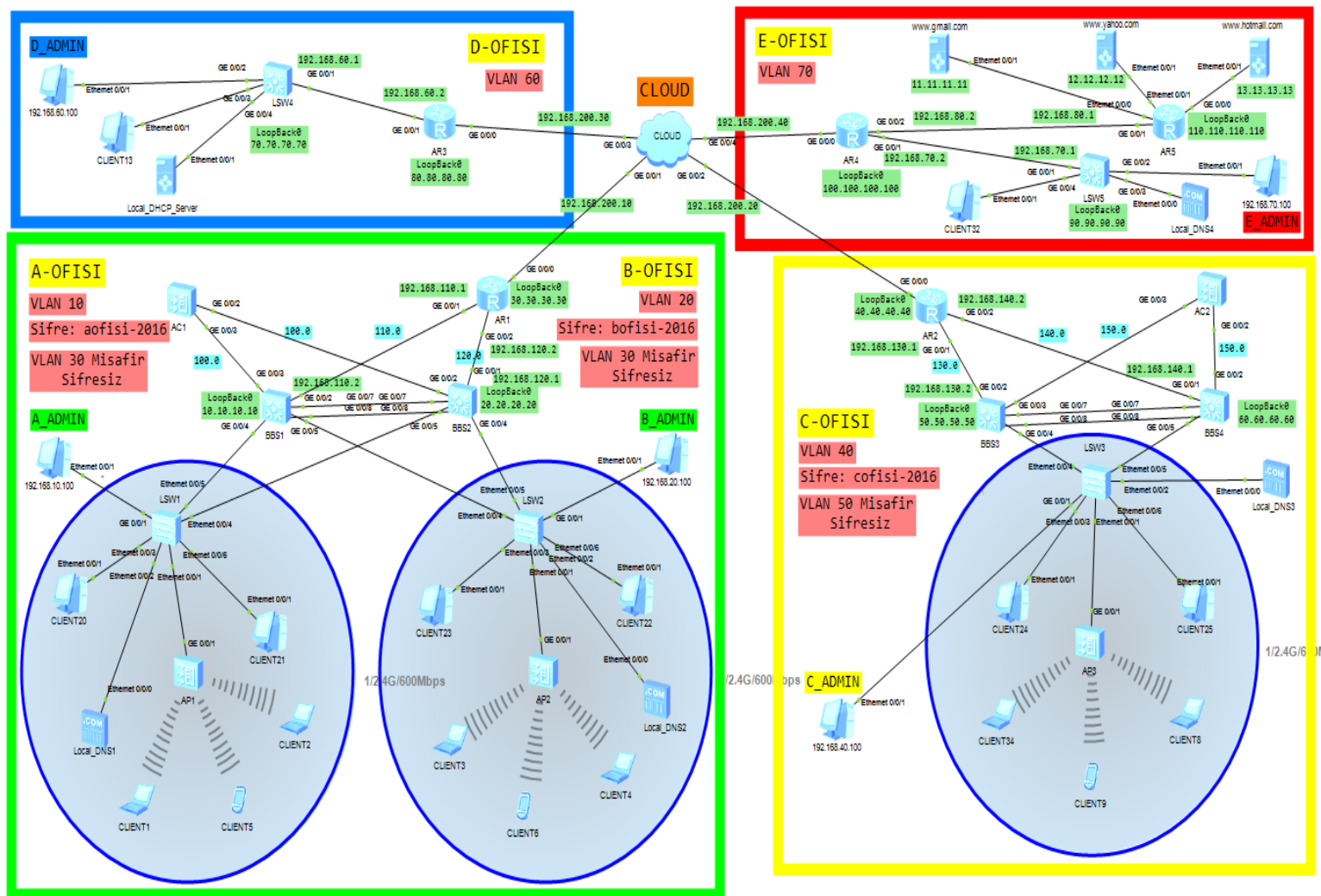

Figure 3

In the first part of the design of the network, all the devices placed and their names were given as shown (Figure 3) in the network map [8].

Then STP (Spanning Tree Protocol) will be applied When STP is implemented measures will be taken to affect performance also other reason that access controller configured with central switches and the design mixed. In the second part of the design, VLANs (Virtual Local Area Network) has created and ip addresses has given as shown in the network map. The main purpose of creating different VLANs for each office is to take communication time against performance degradation. At the same time, if there is an infiltration for any VLAN, only those VLANs can be harmed and this will be very good for security too.

In the third part of the design, one $\mathrm{AC}$ (Access Controller) is connected to the center switches and one AP is added to each side switch. First, the central switches (BBS1, BBS2, BBS3, and BBS4) has configured to $\mathrm{AC} 1$ and $\mathrm{AC} 2$, and the configurations of the ports connecting the edge switches to the APs will be entered. Later, configurations will be made to broadcast on $\mathrm{AC} 1$ and $\mathrm{AC} 2$ devices.

In the fourth part of the design, Local DNSs, admin PCs, wired and wireless devices will be connected. After all of the devices are turned on, for connecting the wireless part of design double click on any of the wireless devices, select the broadcast you want to connect from the pop-up window, and click the Connect button. If the network has password, we should write the password of the selected network correctly and then click on the arrow button to connect to the network. If the network is broadcasting unencrypted, it should be expected to connect to the network only after selecting the broadcast. The WLAN will be entered as the default VLAN from which VLAN you wish to receive IPs from the wired devices that connect to the network and 
connect the wired devices to the edge switches to obtain the correct IP from the DHCP server. Then click twice on any wired device, select the DHCP option from the pop-up window and click the Apply button [9].

In the fifth part of the design, only offices that make cable broadcasts has designed. Routers connected to the switches and switches connected to the PCs the same as A, B and C offices, In the sixth part of the design, the OSPF protocol will be implemented to enable the A, B, C, D and E offices to interact with each other in a performance way, without loss of data.

Once the OSPF(Open short Path First) protocol is configured, all devices will be able to communicate with each other.after connecting all offices with each other Access List can be used to allow or denny any PC or any connection because if all the clients have connection with each other the performance will be down for Example if a user access the A office administrator he can do each configuration and the security also will be down for removing these problems Access list is used in the configuration of network design. As noted above, we must not forget that this design is in virtual labs that are free to share in real lab environments. Because of this, the configurations we can make and the devices we can operate are limited. For accessing the network from far way the necessary configurations are made to enable TELNET connection to the devices [10].

\section{Result}

In this study, LAN, WAN, WLAN networks including routers, switches, hubs and necessary servers, personal computers, phones, DHCP Server, DNS Server are designed and simulated. A packet monitor software simulation was used and the switch were configured with the same device as the actual router in the network topology.

Cisco packet tracer and eNSP emulation programs has been used Instead of using physical devices which allows you to work with multiple network devices in a virtual environment. Along with this there will be a more advantageous work to be done since there will not be wasted time consumed or laboratory to make the cable connections between the laboratory equipment's and much less time is spent on more scenarios with more topology, allowed to run without spending any cost or budget.

In this study, many scenarios that could be applied were given precedents. By increasing these scenarios, it will offer many innovations for network, advanced network, network security and performance is done. Also both static and dynamic IP configurations has been simulated on a work-time basis. The simulation shows that the results on the network can be reached without any problems between a source package and the destination. With Cisco Packet Tracer software, many topologies have been accomplished by performing one real simulation using NS. They have the same configuration as the actual router, switch and Hubs.

Wide networks have been defined and configured on the required devices. For Computers Dynamic Host Configuration Protocol, it has been determined that DHCP pools has been set up and computers accept IP addresses. Also DHCP pool has been created. VoIP operations have been done for IP phones. They have received IP addresses of IP phones and exchanged data with each other. Enhanced Interior Gateway Routing Protocol (EIGRP), static and RIP routing protocols are specified on the Router and it is problematic to check whether the frame relay switch is performing the necessary operations on the router. Network Time Protocol server master and client data center internet network are defined. The DNS server is detected and distributed to the computers through Dynamic Host Configuration Protocol pools.

\section{Referance}

[1]. OLIVIER BONAVENTURE (2015), Computer Networking : Principles, Protocols and Practicehttp://www.computerhope.com/ jargon/n/network.htm.pdf

[2]. JD MEIER (2015) https://www.quora.com/What-is-the-difference-between-performance-testing- load-test.

[3]. İTÜBİDB (2015) Captive Portal (Restriction Portal). İstanbul. http://bidb.itu.edu.tr/seyirdefteri/blog/2013/09/07/captive-portal(k\%C4\%B1s\%C4\%B1tlama-portal\%C4\%B1)

[4]. MEGEP. (2015). Wireless Network Systems. http://www.megep.meb.gov.tr /mte_program_modul/moduller_pdf/Kablosuz\%20A\% C4\%9F\%20Siste

[5]. CISCO (2015) VLANTrunk Protocol. Cisco: http://www.cisco.com/c/en/us/support/docs/lan- switching/vtp/10558-21.html\#req adresinden alındı

[6]. PRESS, C. (2015). Fiber Distributed Data Interface. High-speed computer network: http://www.cisco.com/cpress/cc/td/cpress/ fund/ith2nd/it2408.htm

[7]. HUAWEI. (2015). Feature Description - Security. huawei: http://support.huawei.com/enterprise/docinforeader. action? contentId=DOC0100534396

[8]. SAVAŞAL, S. (2015). Huawei eNSP. Date 6/10/2016 selcuk.savasal: http://www.selcuk.savasal.com/?p=255

[9]. LINKSYS, D. (2015). Linksys configuration. http://www.linksys.com/ca/support-article?articleNum=142912

[10]. BAYDAR, S. (2015). Everything About OSPF. 6 12, 2016 Date: http://www.btyardim.com/ospf-hakkinda-hersey.php 